\title{
USING TIME-VARYING AUTOREGRESSIVE FILTER TO IMPROVE EMG AMPLITUDE ESTIMATOR
}

\author{
Jer-Junn Luh, Guo-Ching Chang, Cheng-Kung Cheng*,Jin-Shin Lai**, Te-Son Kuo \\ Department of Electrical Engineering \\ * Center for Biomedical Engineering \\ **Department of Physical Medicine and Rehabilitation \\ National Taiwan University, Taipei, Taiwan, ROC
}

\begin{abstract}
An electromyography (EMG) amplitude estimator can be the proportional control input of power prostheses or functional electrical stimulation system. In this paper, an improved temporal whitening process through a timevarying autoregressive (AR) filter is evaluated in experiment. The results of our experiment demonstrated time-varying whitening filter can improve the SNR of the amplitude estimators. It implies the influence of nonstationary characteristics of tissue filtering.
\end{abstract}

Keyword: EMG, time-varying autoregressive filter

\section{INTRODUCTION}

Previous studies showed that EMG signals can be modeled as a white noise of which amplitude modulated by muscle force in isometric contraction[1][2]. Therefore, amplitude estimation is useful for muscle force estimation. Whitening is an important technique to improve the SNR of the classical moving-windows-root-mean-square method (MWRMS)[2]. Although all investigators assumed EMG as a wide-sense stationary (WSS) signal , non-stationary characteristic of tissue filtering may influence on amplitude estimation.

In 1989, Moser and Graupe developed a nonstationary model to identify EMG signals as the input commands of a functional electrical stimulation system[3]. We use this time-varying AR filter as a whitening filter to improve SNR of EMG amplitude estimator.

\section{METHODS}

A pair of electrodes was placed on the subjects' muscle belly of right biceps brachii. EMG signals were amplified 1000 times through preamplifier (Gould model 20-4615-58). Torque signal was measured from Cybex 6000 System. All the data were digitized from a 12-bit A/D card (Metrabyte DAS-16) and stored in a 486PC. Subjects were asked for a maximum voluntary contraction (MVC) before test beginning for calibration. In each trial, subjects maintain $50 \%$ or $25 \%$ MVC elbow flexion for 3 seconds at the position of elbow flexion of 60 degrees. The time-varying AR model is described by the following equations[3][4]:

$y(n)=\sum_{i=1}^{k} a_{i}(n) y(n-i)+w(n)$

where $y(n)$ denote the EMG signal at time $n$ and $a_{i}(n)$ is the ith order AR coefficient that evolves under the constraint:

$a_{y}(n)=\sum_{j=1}^{l} b_{i j} a_{i}(n-j)+v_{i}(n)$

where $w(n)$ and $v_{j}(n)$ are mutually orthogonal, zero mean, second moment ergodic white noise processes. We should notice $w(n)$ is the residual of time-varying AR filter which can be the input of MWRMS estimator. The flowchart of signal processing is shown in Fig 1.

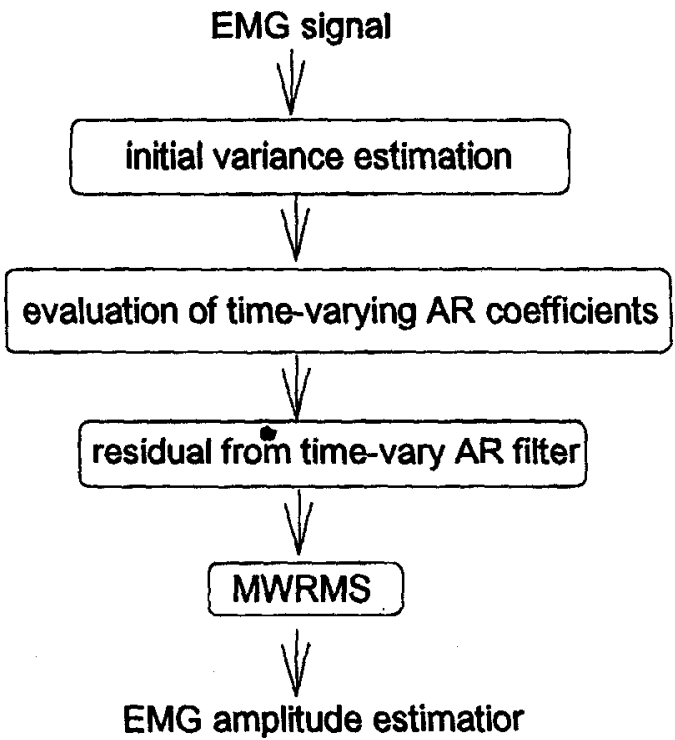

Fig 1 Flowchart of Signal Processing 


\section{RESULTS}

The EMG amplitude from MWRMS without whitening, with linear whitening (modified from [2]), and with timevarying $\mathrm{AR}$ filter whitening are compared. Figure 2 shows a typical EMG data, MWRMS amplitude estimator without whitening, with linear whitening, and time-varying AR filter whitening. Table 1 is the mean and variance of SNR.

Table 1 SNR of EMG Amplitude Estimator

\begin{tabular}{llccc}
\hline & SNR & $\begin{array}{l}\text { without } \\
\text { whitening }\end{array}$ & $\begin{array}{l}\text { with linear } \\
\text { whitening }\end{array}$ & $\begin{array}{l}\text { with time- } \\
\text { varying AR } \\
\text { whitening }\end{array}$ \\
\hline $50 \% \mathrm{MVC}$ & mean & 10.5009 & 10.9323 & 13.4592 \\
& variance & 3.3703 & 4.2510 & 4.3585 \\
\hline $25 \% \mathrm{MVC}$ & mean & 10.2030 & 10.4837 & 11.5953 \\
& variance & 5.1686 & 5.9848 & 15.7385 \\
\hline
\end{tabular}

\section{DISCUSSION}

Initial comparisons among MWRMS EMG amplitude estimators with linear whitening, time-varying AR whitening, and without whitening, the results show that time-varying AR filter can get a better estimation of EMG amplitude. This filter can combine with the estimation of time-vary AR coefficient for power prosthesis control[3]. Time-varying $A R$ filter can eliminate the effect of nonstationary phenomena from muscle to electrode and let residual signal be a white signal, but it may not the inverse of tissue filtering function.

In this experiment, $k=4$ and $l=1$ were choiced for convenience. It can be justified by AIC [4].

\section{CONCLUSION}

The time-varying AR filter technique described herein promises to be a whitening filter that can improve the performance of EMG amplitude estimator. Our result provides an evidence that the influence of non-stationary phenomenon may be an important reason to get a low SNR for non-stationary EMG amplitude estimation.

\section{ACKNOWLEDGMENT}

This research was financially supported by a grant No. NSC 83-0420-B-002-213-M08 from the National Science Council of ROC.

\section{REFERENCE}

[1] N. Hogan and R. W. Mann, "Myoelectric signal processing: optimal estimation applied to electromyogrphay---part $\mathrm{I}$ : derivation of the optimal myoprocessor," IEEE Transaction on Biomedical Engineering, Vol. BME-27, pp.382-395, 1980.
[2] E. A. Clancy and N. Hogan, "Single site electromyograph amplitude estimation," IEEE Transaction on Biomedical Engineering. Vol. 41, pp.159-166, 1994.

[3] A. T. Moser and D Graupe, "Identification of nonstationary models with application to myoelectric signals for controlling electrical stimulation of paraplegics," IEEE Transaction on Acoustics, Speech, and Signal Processing. Vol 37, pp.713-719, 1989.

[4] G. Kitagawa and W. Gersch, "A smothness priors timevarying AR coefficient modeling of nonstationary covariance time series," IEEE Transaction on Automatic Control, Vol. AC-30, pp.48-56, 1985.
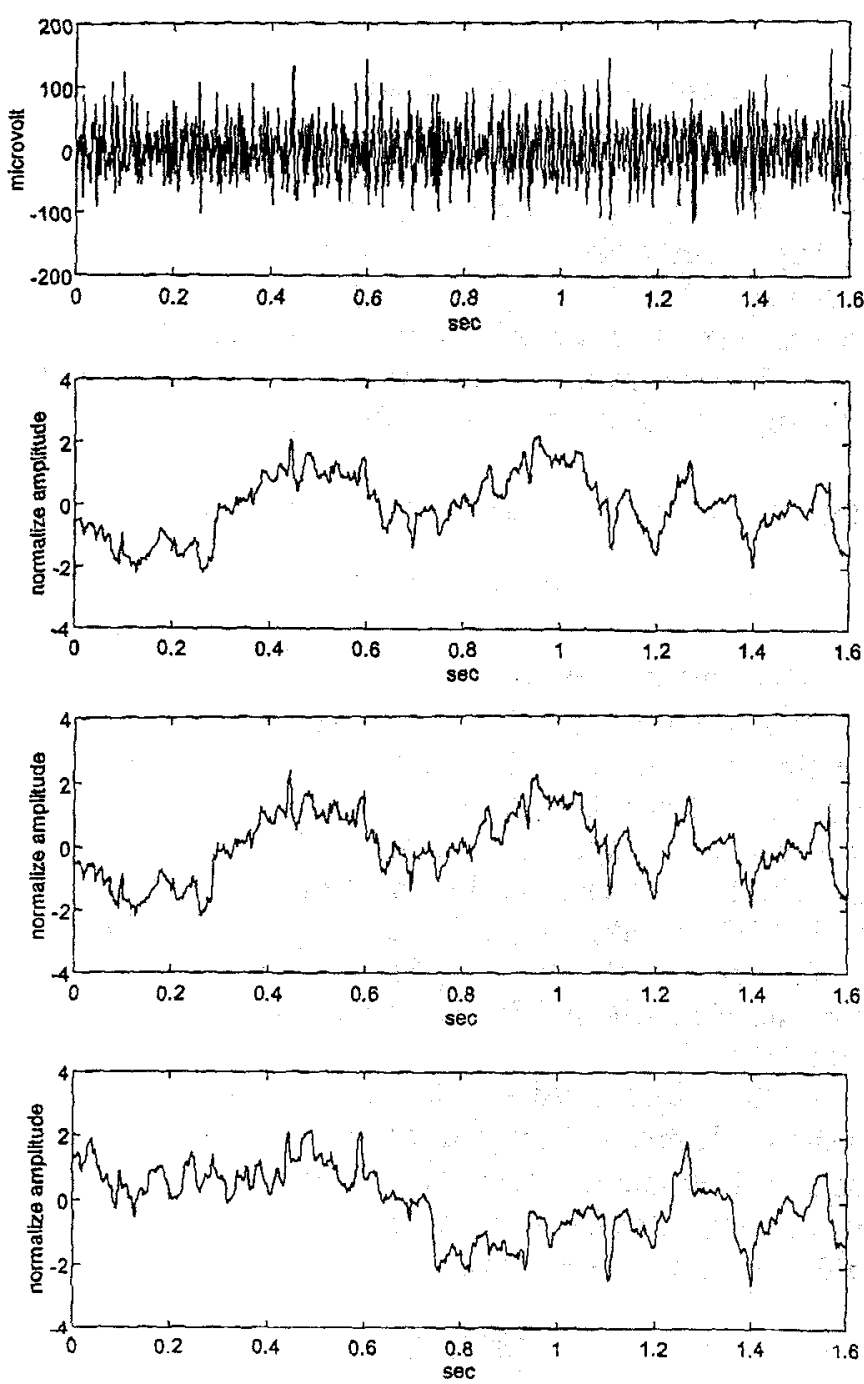

Fig2 Typical MWRMS EMG amplitude estimator. From top to dowm: original EMG signal, amplitude estimator without whitening ( $S N R=13.21$ ), amplitude estimator with linear whitening ( $\mathrm{SNR}=13.79)$, and amplitude estimator with timevarying AR filter whitening ( $S N R=17.5194)$ 